\title{
Trsper: a web-based application for Archimedes spiral analysis
}

\author{
Rogan Magee^, Benjamin Yang, Jeff Ratliff \\ Department of Neurology, Sidney Kidney Medical College at Thomas Jefferson University, Philadelphia, PA, USA \\ Contributions: (I) Conception and design: All authors; (II) Administrative support: All authors; (III) Provision of study materials or patients: All \\ authors; (IV) Collection and assembly of data: All authors; (V) Data analysis and interpretation: All authors; (VI) Manuscript writing: All authors; (VII) \\ Final approval of manuscript: All authors. \\ Correspondence to: Rogan Magee. Hospital of the University of Pennsylvania, 3400 Spruce Street, Philadelphia, PA 19104, USA. \\ Email: rgmcds@gmail.com.
}

\begin{abstract}
Background: We built a web-based application of the Archimedes spiral exam that implements clinically validated spiral metrics and tested drawing instructions to define a clinical workflow.

Methods: We designed an HTML5 and Javascript implementation of the spiral exam to run on mobile touchscreen devices. We then recruited 10 volunteers each for 2 experiments designed to validate the programmed spiral metrics and assess how instructions or drawing implement affect the results. In task one, volunteers drew 5 spirals each while following 6 different instruction sets $(n=30$ spirals each, $n=300$ spirals total) that varied by support of the drawing hand and tracing condition (either tracing a spiral template, drawing in-between it, or freehand). In task two, volunteers drew 5 spirals each while following 2 instruction sets and drawing using a stylus or their dominant index finger ( $n=20$ spirals each, $n=200$ spirals total).

Results: Principal components analysis of calculated metrics revealed that the experiments grouped by instruction set and by subject. Mean Euclidean distance between experiments represented as 11-dimensional vectors revealed that consistency varied among instruction tasks and that drawing with a stylus produced more consistent results than did using the dominant index finger. Using experimental data and simulated abnormal spirals, we designed a decision support system that accurately identifies potentially abnormal spirals.
\end{abstract}

Conclusions: We built and validated a robust digital implementation of the Archimedes spiral exam and recommend a sensitive and specific workflow on the basis of data gathered from healthy volunteers.

Keywords: Archimedes spiral; movement disorders; tremor; mobile device; web application

Received: 05 April 2021; Accepted: 15 July 2021; Published: 20 January 2022.

doi: $10.21037 /$ mhealth-21-16

View this article at: https://dx.doi.org/10.21037/mhealth-21-16

\section{Introduction}

Archimedes spiral drawing is a cornerstone of the movement disorders exam. Clinically validated, automatic analysis metrics have been developed, including: frequency (1-4) and spiral axis in essential tremor (ET) (5) and simulated dystonic tremor (6); loop width variation in ET (7), Niemann-Pick disease type C (8), Parkinson's disease (9), multiple sclerosis, and functional tremor $(10)$; and velocity $(11,12)$. Studies investigating these metrics have digitized the spiral exam through scanned paper drawings (13-15) or Wacom tablet applications, which capture pen strokes from a pen-and-paper drawing (2,3,16-18). NeuroGlyphics (2) (http://neuroglyphics. org/) and MovAlyzeR (http://neuroscript.net/) are example Wacom programs. Other applications for personal digital assistants (19-21), iPad (22), and mobile phone have been developed (23). The effects of drawing instructions on outcomes, e.g., comparing tracing a template to drawing freehand, have also been explored (24). Notably, the group found that different drawing instructions affected ratings of spiral severity.

To increase access to a digital exam format, we built a

^ ORCID: 0000-0003-3899-2140. 


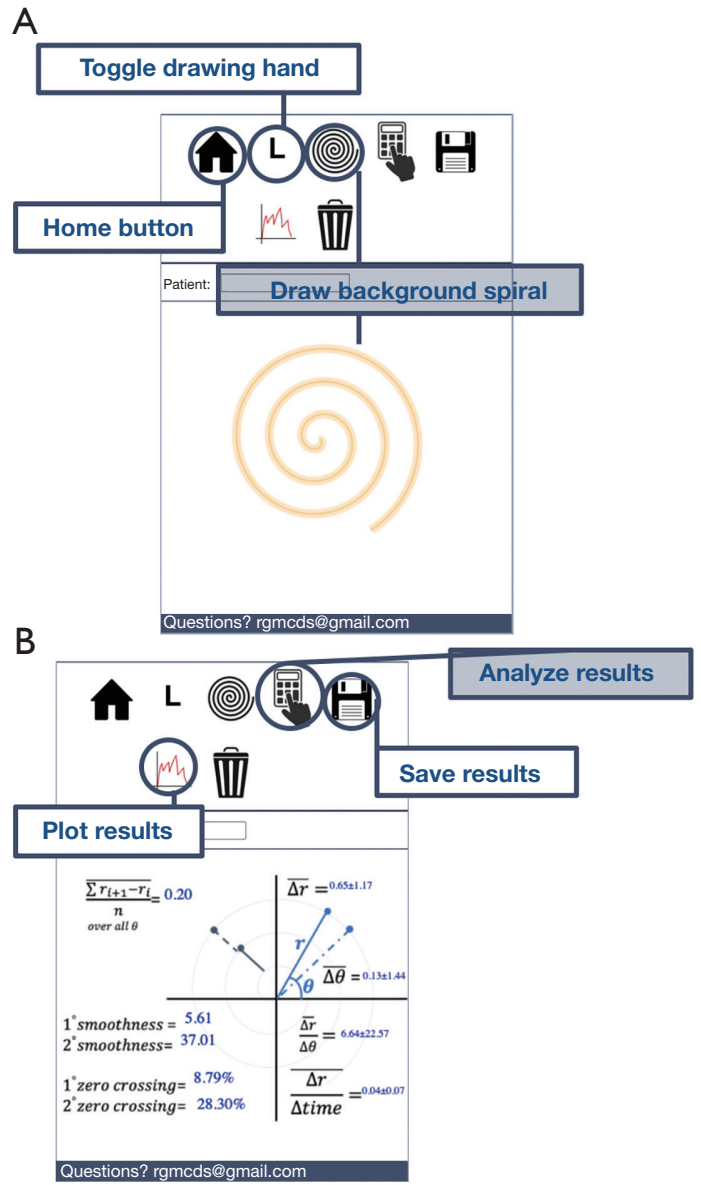

Figure 1 Demonstration of web application. (A) A screen capture of the main spiral drawing task, highlighting buttons for: homereturn to the home page, toggle- to record which hand was used to draw the spiral, background-a button to bring up a background ideal spiral in orange relief. Clicking the last button up to 5 times increases the background spiral size. (B) A screen capture of the main spiral drawing task, highlighting buttons for: analysis-to analyze the drawn spiral and bring up a visual representation of calculated metrics, save-to save the results, and plot-to plot all saved results under the chosen "patient name".

web-based platform for mobile touchscreen devices that incorporates clinically validated metrics, such as $1^{\text {st }}$ and $2^{\text {nd }}$ order smoothness (25). Then, we assessed in-application characterization of spirals drawn by healthy volunteers following varying instruction sets (24) and compared consistency across instruction sets and individuals. Our analysis reveals that instruction set and drawing implement affect spiral metrics. These experiments introduce a tool and set of instructions for its use that may afford greater sensitivity and specificity for detection of pathology through Archimedes spiral drawing. In addition, as our web-based platform can be accessed freely, remotely, and without the requirement for any specialized equipment beyond consumer grade tablets or smart phones, it also has the advantage of lowering economic and physical barriers to testing. Future validation experiments will evaluate the application's performance in identification of abnormal movements in diverse patient populations (e.g., those with dystonia, tremor, myoclonus) and help us to amend and expand the tool to better characterize those abnormalities. Further experiments will also expand our understanding of how well the application tracks changes in movements caused by disease progression or by normal aging. We present the following article in accordance with the MDAR reporting checklist (available at https://dx.doi.org/10.21037/mhealth-21-16).

\section{Methods}

\section{Web application design}

The web application for Archimedes spiral analysis was written using HTML5 and vanilla Javascript code (http://www.trsper. com) (Figure 1). The website will remain freely available, and the code is open source and available for download. For the background spiral, simulated ideal Archimedean spiral Cartesian coordinates are calculated according to the formula: $x=(v t+c) \cos w t, y=(v t+c) \sin w t$, where $t$ represents time and $w$ and $v$ are constants reflecting the angular velocity of the spiral's rotation about the origin. Users draw in the main canvas and all touch points are stored locally as $\mathrm{x}, \mathrm{y}$ coordinates and time, then drawn as a blue line with a refresh rate of $50 \mathrm{~Hz}$.

\section{Participants}

Volunteers were recruited through emails to Thomas Jefferson University students. All volunteers were putatively healthy, equally distributed by gender (task one: 4 female, 6 male; task two: 6 female, 4 male), and spanned ages 28 to 55 . All participants were informed of the purpose of the study and consented for participation. The study was conducted in accordance with the Declaration of Helsinki (as revised in 2013). The Thomas Jefferson University Institutional Review Board reviewed and exempted the study (Control \#20E.990).

\section{Spiral drawing tasks}

Ten distinct volunteers each were recruited for two separate 

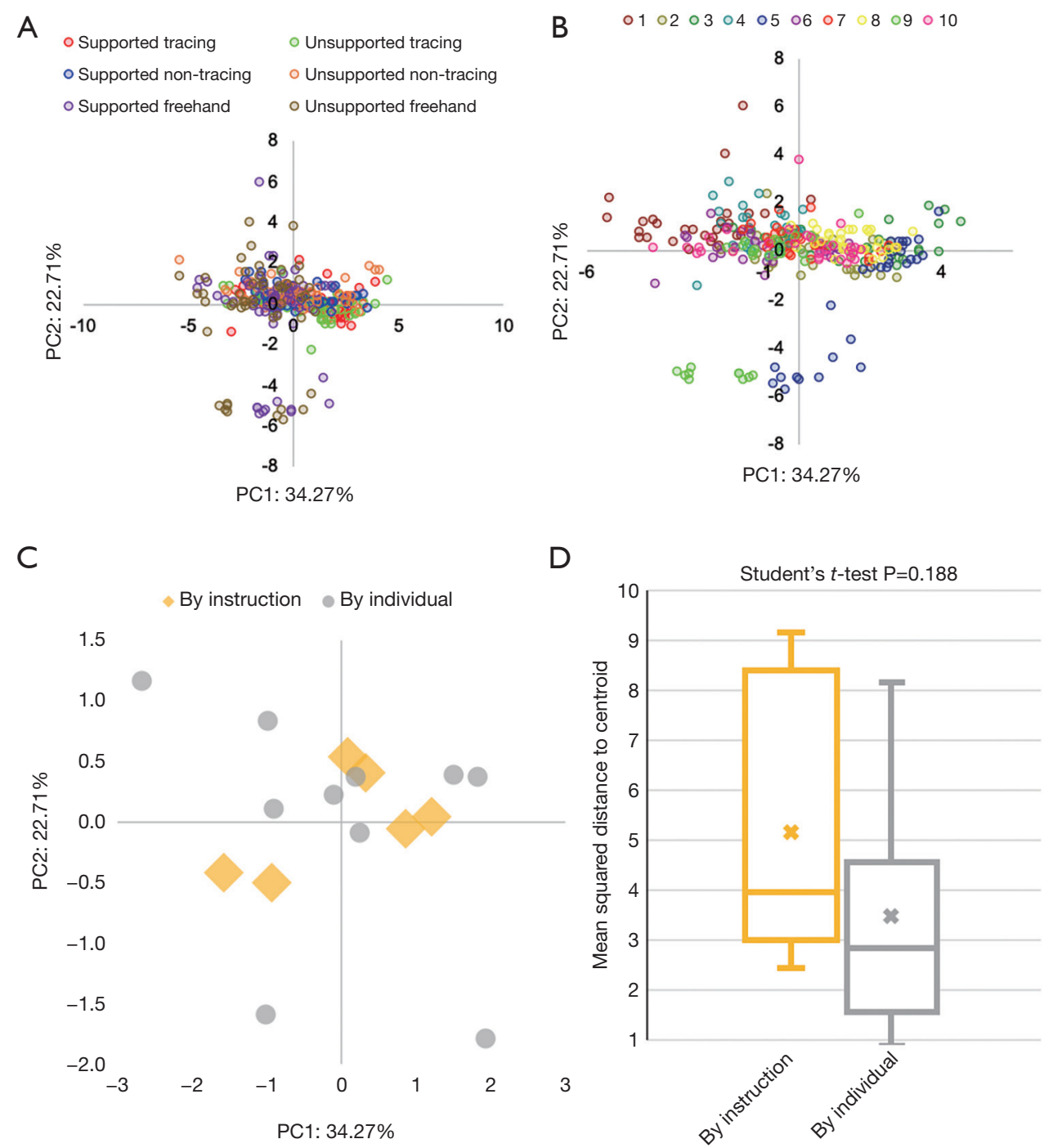

Figure 2 Task one or analysis of spiral metrics by instruction set. (A) Principal components analysis (PCA) of the 11 metrics calculated for each of the 300 spirals from task one reveals grouping by each of six instruction sets: red—supported tracing, green—unsupported tracing, blue-supported non-tracing, orange-unsupported non-tracing, purple-supported freehand, brown-unsupported freehand. We plot each set of 11 metrics by the first and second principal components of the PCA transform. X and Y-axes are labeled with the percentage of variance explained by each principal component. (B) Re-colored PCA by experimental subject again indicates that spirals group by subject of origin. (C) K-means clustering of PCA by instruction set (gold diamonds) and by subject (grey circles) reveals separation of data point by instruction set and subject. Mean distance between centers by instruction set (1.441) is lower than mean distance by subject (2.213) (Student's $t$-test $\mathrm{P}$ value $=0.006$ ). (D) Mean squared distance to cluster centroid for all 6 clusters by instruction set (5.141) and all 10 clusters by subject (3.463) differs significantly (Student's $t$-test $\mathrm{P}$ value $=0.188)$.

tasks. In one (Figure 2), volunteers drew five spirals each according to six previously described instruction sets (24) ( $\mathrm{n}=30$ per person). They used a passive stylus and an iPad. Briefly, instructions were: tracing-trace the background spiral, non-tracing-draw between the lines of it, or draw freehand, while either resting their drawing arm/ hand comfortably on the table or lifting it and allowing no contact between their entire arm and the table. When drawing "freehand", users were asked to recreate the size and shape of the background spiral that they previously 


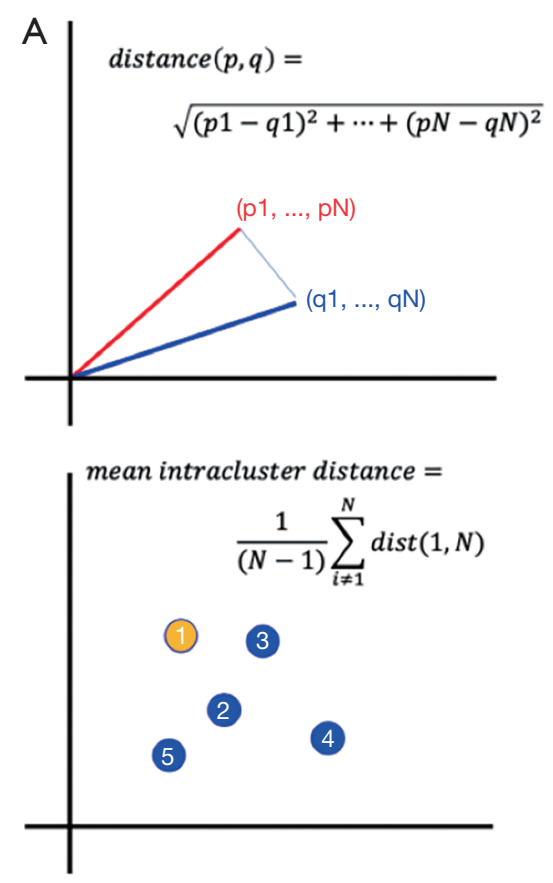

B

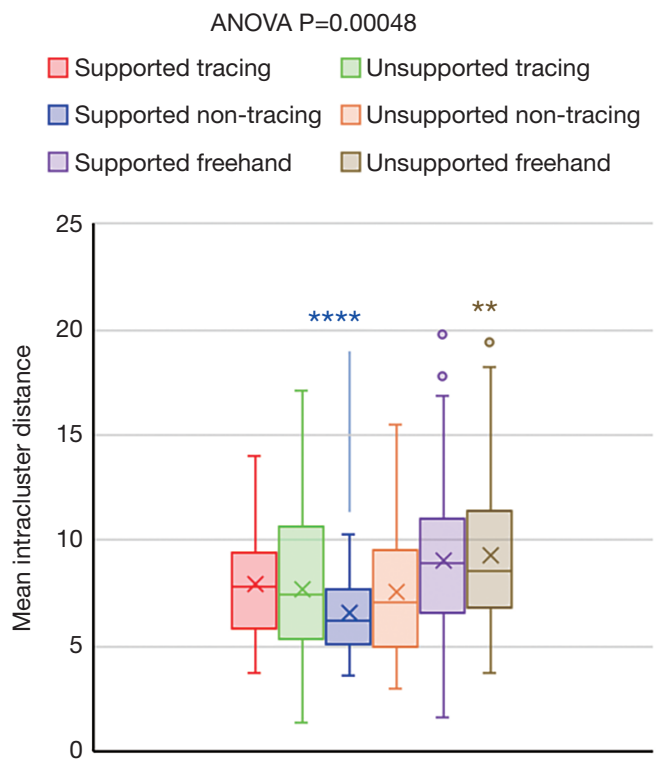

Figure 3 Task one or analysis of spiral metrics by instruction set. (A) A visual representation of the Euclidean distance metric between two experiments represented as N-dimensional vectors and our "mean intracluster distance" metric, calculated as the mean of the Euclidean distance from 1 point in a set of 5 replicates from a single instruction set and single subject to the other 4. (B) Boxplot showing the mean intracluster distance calculated for all of the points in a single set of replicates, grouped by instruction set. ANOVA reveals statistical difference in comparison of all 6 groups $(\mathrm{P}=0.00048)$ and one-vs.-all $t$-test reveals significant difference between the resting between instruction set and all others $\left(* *, \mathrm{P}<0.01 ;{ }^{* * *}, \mathrm{P}<0.0001\right)$.

saw to the best of their ability. We then analyzed each spiral according to the metrics described below in order to determine how the instructions affected the consistency of drawn spirals (Figure 3). In task two (Figure 4), volunteers drew five spirals each using both a passive stylus and their dominant index finger, according to the two most consistent instruction sets as determined in task one (Figure 3D).

\section{Spiral metrics}

Spiral metrics were adapted from prior publications and included:

$$
\begin{aligned}
& \text { (I) } \Delta r=\left(r_{i+1}-r_{i}\right) \text { for } \mathrm{i}=0 \text { to } \mathrm{N} \text { (3) } \\
& \text { (II) } \Delta \theta=\left(\theta_{i+1}-\theta_{i}\right) \text { for } \mathrm{i}=0 \text { to } \mathrm{N} \text { (3) }
\end{aligned}
$$

(III) $\frac{\Delta r}{\Delta \theta}=\frac{\left(r_{i+1}-r_{i}\right)}{\left(\theta_{i+1}-r_{i}\right)}$ for $\mathrm{i}=0$ to $\mathrm{N}$ (3)

(IV) $\frac{\Delta r}{\Delta t}=\frac{\left(r_{i+1}-r_{i}\right)}{\left(t_{i+1}-t_{i}\right)}$ for $\mathrm{i}=0$ to $\mathrm{N}(3)$

(V)

$$
R M S_{\Delta r}=\sqrt{\sum\left(\Delta r_{i}-\overline{\Delta r}\right)^{2}}
$$
$\overline{S W V I_{\theta}}=\sum_{0}^{\theta} \frac{\sigma_{r}}{\mu_{r}}$ or the average coefficient of variation of given spiral widths through all angles 0 to 360 (7)

$$
\begin{aligned}
& \text { (VII) } 1^{\circ} \text { smoothness }=\ln \left(\frac{1}{\theta} \Sigma\left(\frac{\Delta r}{\Delta \theta}-\overline{r_{\theta}}\right)^{2}\right) \\
& \text { (VIII) } 2^{\circ} \text { smoothness }=\ln \left(\frac{1}{\theta} \Sigma\left(\frac{\Delta \frac{\Delta r}{\Delta \theta}}{\Delta \theta}-\Delta \overline{r_{\theta}}\right)^{2}\right)
\end{aligned}
$$


A
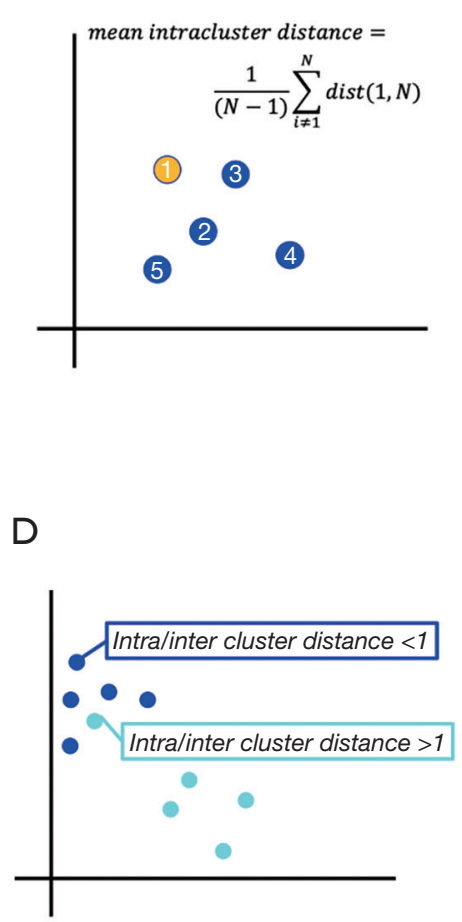

\section{B}

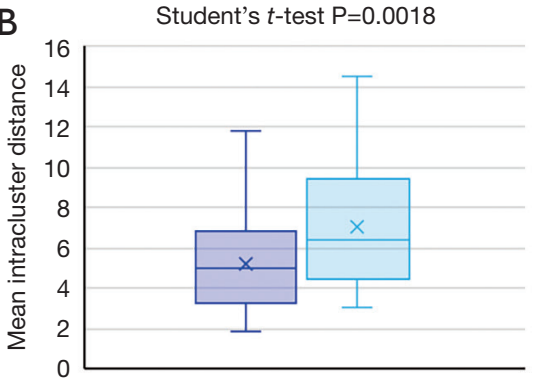

$\square$ Supported non-tracing stylus

$\square$ Supported non-tracing finger

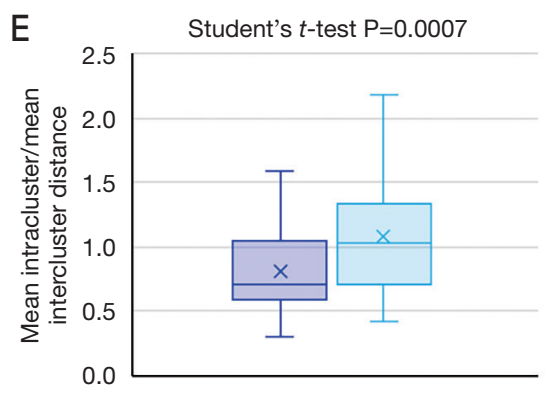

$\square$ Supported non-tracing stylus

$\square$ Supported non-tracing finger

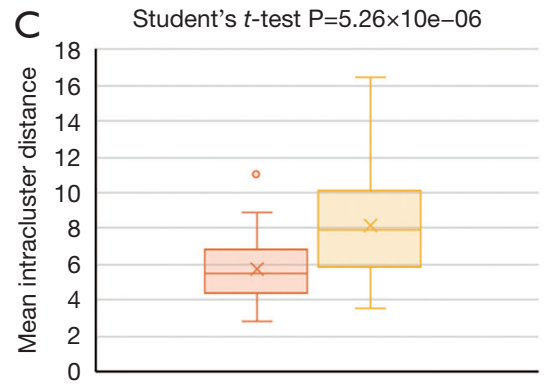

$\square$ Unsupported non-tracing stylus

$\square$ Unsupported non-tracing finger

F

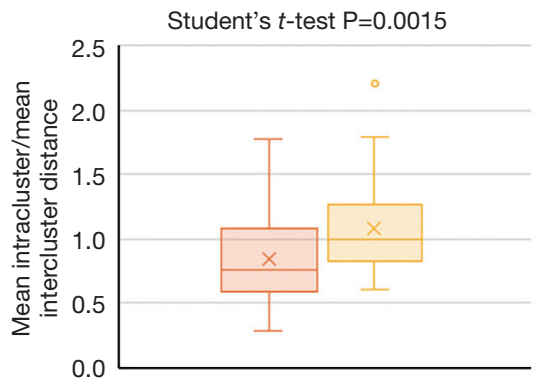

$\square$ Unsupported non-tracing stylus $\square$ Unsupported non-tracing finger

Figure 4 Task two or analysis of spiral metrics by drawing implement. (A) A visual representation of the "mean intracluster distance" metric, as in Figure 3A. (B) Boxplot of the mean intracluster distance for all experiments in the supported non-tracing task comparing drawing with a stylus to drawing with a finger. Student's $t$-test reveals a significant difference $(\mathrm{P}=0.0018)$ between the groups. (C) Boxplot of the mean intracluster distance for all experiments in the unsupported non-tracing task comparing drawing with a stylus to drawing with a finger. Student's $t$-test reveals a significant difference $\left(\mathrm{P}=5.26 \times 10^{-6}\right)$ between the groups. Outliers are marked with individual data points. (D) Visual representation of the expected ratio of mean intracluster to mean intercluster distance. Intracluster distance is the mean Euclidean distance between 1 point in a set of replicates of the same instruction set from the same subject to all 4 other points within the group. Intercluster distance is the mean distance between a point in one set of replicates and the points in the alternate set of replicates, e.g., a point in the supported non-tracing, stylus drawn spirals from one subject to the points in the supported non-tracing, finger drawn spirals from the same subject. (E) Boxplot of the ratio of mean intracluster to mean intercluster distance for all experiments in the supported non-tracing task comparing drawing with a stylus to drawing with a finger. Student's $t$-test reveals significant difference $(\mathrm{P}=0.0007)$ between the groups. (F) Boxplot of the ratio of mean intracluster to mean intercluster distance for all experiments in the unsupported non-tracing task comparing drawing with a stylus to drawing with a finger. Student's $t$-test reveals significant difference $(\mathrm{P}=0.0015)$ between the groups. Outliers are marked with individual data points.

$$
\begin{aligned}
1^{\circ} \text { zero crossing } & =\left[\frac{1}{2(N-1)} \sum \operatorname{sign}\left(\frac{\Delta r}{\Delta \theta_{i+1}}-\overline{r_{\theta}}\right)-\operatorname{sign}\left(\frac{\Delta r}{\Delta \theta_{i}}-\overline{r_{\theta}}\right)\right] \times 100 \% \\
2^{\circ} \text { zero crossing } & =\left[\frac{1}{2(N-1)} \sum \operatorname{sign}\left(\frac{\Delta \frac{\Delta r}{\Delta \theta}}{\Delta \theta_{i+1}}-\overline{r_{\theta}}\right)-\operatorname{sign}\left(\frac{\Delta \frac{\Delta r}{\Delta \theta}}{\Delta \theta_{i}}-\overline{r_{\theta}}\right)\right] \times 100 \%
\end{aligned}
$$




\section{Simulated spirals and decision support}

To build decision support, we simulated "abnormal" spirals by first calculating an ideal Archimedes spiral, dithering every point by 4 pixels in either $\mathrm{x}$ or $\mathrm{y}$ direction with $50 \%$ probability, and then adding noise from an ideal cosine function to create 9 distinct spiral types 1 through 9 (Figure 5A). For types 1 through 4, cosine noise was added to only 1 of the 4 spiral quadrants. For type 5 , cosine noise was added to all 4 quadrants which was then doubled and changed every 5 points to generate types 6 and 7 . Similarly, types 8 and 9 were generated using the same method as types 6 and 7, but only half the amount of cosine noise was added to each quadrant.

We simulated each of the 10 spiral types 2,000 times, at each of 20 possible sizes. We then found the centroid (Figure S1A) of each of the 2,000 simulations, resulting in 200 total centroids. Next, we calculated the distance between the drawn spiral and the control or type 0 spiral for each of the 20 possible sizes, as well as the closest centroid for the 9 types of abnormal spirals in that size. Finally, we calculate a ratio of these distances for each of the 20 possible sizes. We then report the 5 ratios closest to the size of the drawn spiral, by comparing the area of the drawn spiral (calculated using $x$ and $y$ bounds of the spiral) to each of the 20 possible sizes we simulated.

\section{Statistical analysis}

All spiral coordinates were first transformed from Cartesian to polar coordinates, following the formulae: $r=\sqrt{x^{2}+y^{2}}$ and $\theta=\arctan \left(\frac{y}{x}\right)$ where theta was computed in radians and the values adjusted so they continuously increased from zero, as previously described (25). We represented each individual experiment as an $n$-dimensional vector of spiral metrics. Principal components analysis (PCA) transformed high-dimensional data into lower dimensions that preserved variability and allowed for visualization of relationships across conditions. K-means clustering quantified relative relationships of PCA groups. For each group of 5 technical replicates, we then calculated the distance between an experiment and each of the 4 other members of its group using Euclidean distance: $\sqrt{\left(p_{1}-q_{1}\right)^{2}+\cdots+\left(p_{n}-q_{n}\right)^{2}}$ (Figure 2A). We used ANOVA and one-vs.-all $t$-tests to compare this calculated metric in our experiments. All original data were included in the study. All data were normally distributed according to a KolmogorovSmirnov test.

\section{Results}

To validate our spiral drawing application (Figure 1), we tested the effect of distinct drawing instructions that practicing neurologists might give patients (see Methods).

\section{$P C A$}

We visualized our data using PCA, which reduces data dimensions while preserving variability. PCA of task one data with all 11 metrics and coloring by instruction set reveals outliers in each freehand instruction set (Figure 2A), as well as clustering by instruction set. Coloring observations by subject rather than by instruction set also reveals outliers (Figure 2B) and clustering by subject.

$\mathrm{K}$-means clustering to determine the center of each of these clusters (Figure 2C) in the PCA transformation reveals a significant increase in the distance between centers, comparing six instruction sets (mean $=1.441$ ) to the ten subjects (mean $=2.213$ ) (Student's $t$-test $\mathrm{P}$ value $=0.006$ ). The mean squared distance of points to the cluster centroid is higher when clustering by the six instruction sets (mean $=5.141$ ) as compared to clustering by the ten subjects (mean $=3.463$ ), although this difference is not statistically significant (Student's $t$-test $\mathrm{P}$ value $=0.188$ ) (Figure 2D). These results suggest there was more variation in the 11 calculated metrics between subjects than between instructions.

\section{Intracluster distance by instruction set and individual}

We next investigated the effect of drawing instructions on the consistency of spiral metrics from each individual, by calculating the mean Euclidean distance (Figure 3A) between each replicate (represented as 11-dimensional vectors of spiral metrics) and the other 4 replicates in their experimental group (dubbed "mean intracluster distance"). The mean Euclidean intracluster distance differed significantly across the six instruction sets (ANOVA $\mathrm{P}$ value $=4.8 \times 10^{-4} ;$ Figure $3 B$ ), similarly to observer-rated tremor severity in a prior study of the effect of drawing instructions on hand-drawn spirals. Notably, supported non-tracing differed significantly from all other groups (one-vs.-all Student's $t$-test $\mathrm{P}$ value $<0.0001$; Figure $3 B$ ), as did unsupported freehand (one-vs.-all Student's $t$-test $\mathrm{P}$ value $<0.005 ;$ Figure $3 B$ ). As a result, we concluded that the supported non-tracing instruction set produces drawings with the most consistent spiral metrics, while the 

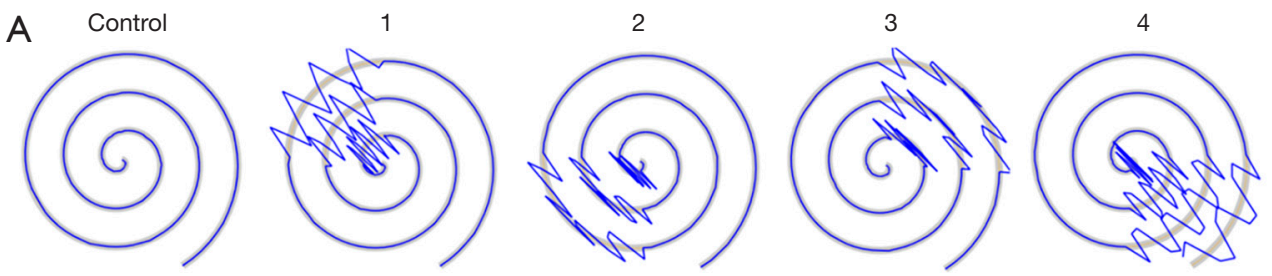

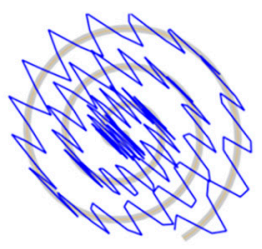

5

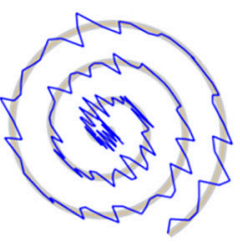

6

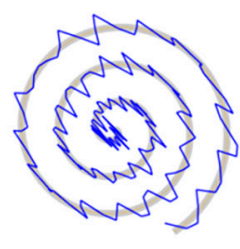

7

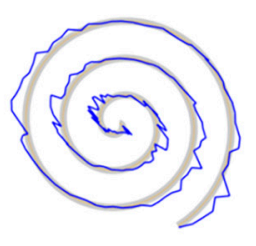

8

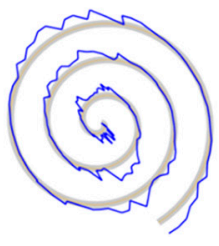

9
B

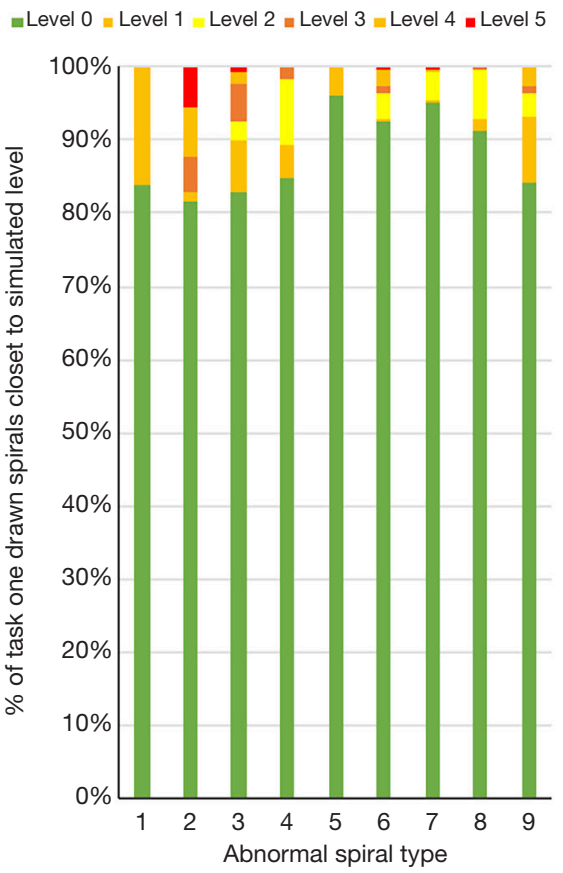

C

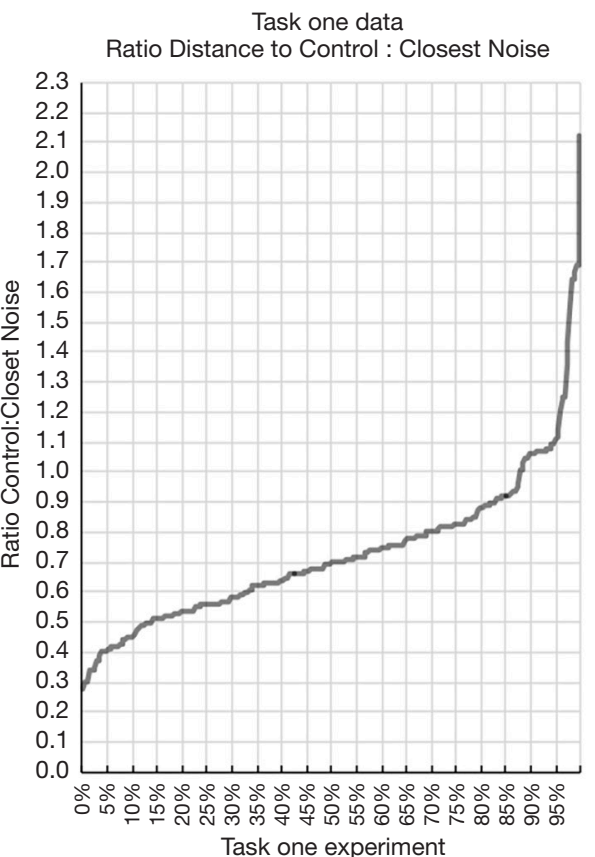

Figure 5 Spiral simulations and decision support by Euclidean distance. (A) We show 10 examples of simulated spirals, including a control spiral with slightly dithered points from a perfect Archimedean spiral, and 9 types of noisy spirals. (B) We simulated 1,000 spirals each over 5 different levels of noise, for each of the 9 types of noise and collected the centroids of each simulated set. We then asked which level of noise each of the 300 real spirals from task one was closest to by Euclidean distance (as in prior tasks, the distance between the metric vectors for real data and centroid), including level 0 or control or no noise. We then show what percentage of each of the 300 spirals was closest to each level, for the 9 types of noise we plotted. (C) Finally, we computed the ratio of the distance between the real data and the control centroid over the distance between real data and the next closest noise centroid, for each of 20 different sizes of ideal Archimedean spiral. We sort these ratios in order and retain the lowest 5 and then plot the median value for each of the 300 real experimental samples. 
unsupported freehand instruction set produces drawings with the least consistent spiral metrics.

\section{Intracluster and intercluster distance of finger or stylus drawn spirals}

For task two (Figure 4), we examined the effects of drawing with a stylus as compared to drawing with a finger. For comparison, we calculated the ratio of mean intracluster over mean intercluster distance (Figure $4 D$ ), where a ratio $>1$ indicates a point that is closer to the points in the opposite group, while a ratio $<1$ indicates a point that is closer to points within the same group.

When comparing stylus drawings to finger drawings individually in each instruction set, we found lower mean intracluster distances (Figure 4A) for stylus drawn spirals in both the supported non-tracing (mean 5.21 vs. 6.99; Student's $t$-test $\mathrm{P}$ value $=0.0018$; Figure $4 B$ ) and unsupported non-tracing (5.72 vs. 8.09; Student's $t$-test $\mathrm{P}$ value $=5.26 \times 10^{-6}$; Figure $4 C$ ) instruction sets, suggesting stylus drawn spirals are more consistent than finger drawn spirals in these instruction sets. Consistent with these results, we also found lower mean ratios of mean intra/inter-cluster distances (Figure $4 D$ ) in stylus drawings for both supported non-tracing $(0.81$ vs. 1.08; Student's $t$-test $\mathrm{P}$ value $=0.0007$; Figure $4 E)$ and unsupported non-tracing (0.84 vs. 1.09; Student's $t$-test $\mathrm{P}$ value $=0.0015$; Figure $4 F$ ). Together, these results suggest that drawing with a stylus produced more consistent spirals than drawing with the dominant index finger.

\section{Decision support}

We next tried to determine whether we could generate abnormal spirals mathematically by introducing tremulous artefacts into simulated spiral drawings. We first simulated control spirals and then simulated 9 different types of abnormal spiral (Figure 5A). For each of the 9 types, we simulated $n=2,000$ spirals at 6 different "levels", with level 0 being a control or normal spiral and each subsequent level combining a higher amplitude of abnormal spiral with the control template. To assess whether our abnormal simulated spirals differed substantially from drawn control spirals, we calculated the distance between all drawn spirals in task 1 and the centroids of both the abnormal simulated spirals and control spirals. Using these distances, we classified each drawn spiral as either being closer to the control spiral or one of five levels of simulated abnormal spirals. As one example, $84 \%$ of drawn spirals were closer to control than any of the levels of the type 1 spiral (Figure 5B). As expected, most drawn spirals clustered with the control type spirals, regardless of simulated spiral type (Figure 5B). Thus, our mathematically simulated abnormal spirals can be accurately differentiated from spirals drawn by healthy volunteers.

To determine the appropriate number of simulations for use in a decision support system, we then simulated ten groups of control ideal Archimedean spirals with $\mathrm{n}=10,50,100,250$, $500,750,1,000,2,000,5,000$, and 10,000. To analyze each individual spirals against each other, we computed centroids (Figure S1A) based on PCA. We calculated spiral metrics for these 10 groups and plotted both a PCA (Figure S1B), as well as distance to the PCA centroid (Figure S1C). Of note, distance to the centroid plateaued above $n=2,000$ simulations, indicating that additional spiral simulations did not contribute new information.

Finally, we calculated the ratio of distance to "control" simulation over distance to the nearest abnormal spiral of any of 9 types, for all 300 spirals from task one and for all sizes of simulated spiral $(\mathrm{n}=20)$. We then plot the minima for all 300 drawn spirals in ascending order, with range 0.28 to 2.73 (Figure 5C). Ninety-five percent of all drawn spirals showed ratio $<1.1$, indicating that the majority of drawn spirals were closer to control simulation than the most comparable abnormal simulation. Thus, we take all values $>1.1$ to be considered "abnormal" in our decision support system.

\section{Discussion}

We developed a web-based, touchscreen application for the Archimedes spiral drawing task that implements validated metrics for spiral analysis. We then conducted experiments with healthy volunteers to determine the application's discriminatory capacity. We analyzed a potential workflow using this application into our experiments, asking volunteers to follow six different published instruction sets-task one, and to draw with a stylus or their dominant index finger-task two. Analysis of data from task one suggested that more variability between the individual experiments is explained by individual subject rather than instruction set used. In turn, these observations suggest that these metrics capture true biological variability, because the metrics were more sensitive to who was drawing the spirals than how they drew them.

Assessing the instruction sets using Euclidean distance showed that supported non-tracing drawings and 
unsupported freehand drawings produced the most and least consistent replicates, respectively. Prior work with these instruction sets found that supported drawings were rated less severely than unsupported drawings, but showed lower intra- and inter-rater reliability (24). Due to the inherent difference in these tasks with regard to comfort and sensory cues, this result is biologically sound and supports our hypothesis that drawing task affects spiral consistency. We reason that these results may affect sensitivity and specificity of clinically validated metrics like spiral width variability $(7,10)$ as they capture disease correlates. As drawing unsupported and freehand strips away visual and physical compensations for tremor, these instruction sets may be the most sensitive for capturing abnormalities. Conversely, drawing supported and non-tracing may be most specific, given higher inherent consistency.

We also simulated abnormal spirals that vary in severity and were designed to simulated tremulous oscillations. As expected, these abnormal spirals were distinguishable from the drawn spirals of healthy volunteers (Figure 5B,5C). Nontremulous disorders of motor control (e.g., bradykinesia) may not be as readily captured by this system. Future work will look to adequately simulate these conditions, in order to improve their identification using this application.

We also observed that the choice of stylus or finger did affect the consistency of spiral metrics. As a result, we caution users to interpret results according to the drawing implement used. Further, as with the instruction sets discussed above, we note that use of stylus could provide more precise and specific results, and that using a finger could provide more sensitive results.

Implementing this application as a web-based platform provides great flexibility. Users need not download the application. They can instead navigate to a website on any WiFi-enabled touchscreen device. Given the recent expansion of teleneurology, this and similar applications may support the push to integrate smartphones and sensors to capture high quality, remote and objective data (26).

Potential limitations include the varying technical characteristics of accessing devices. Prior studies employed Wacom tablets, which record drawing pressure and provide unparalleled accuracy in stylus position. There may be inherent differences in the sensitivity of Mac or Android devices to stylus drawing or finger drawing. Because technical specifications are not public, we are unable to comment on device differences, but instead note this limitation and urge users to interpret their results with caution when moving between devices. Additionally, our application is limited by design to only capture coordinates in the 2D plane. Wacom applications capture pressure and $\mathrm{Z}$-axis deviations, but this capacity is limited as increasing tremor severity causes users to lift their styli. Future work will determine how well our application handles such situations. Future experiments will also establish how well this application identifies abnormal movements in specific diverse patient populations, for example, in parsing those with tremor due to dystonia from those with tremor due to ET. We will also seek to understand how spiral drawing performance changes with normal aging and with disease progression, with an eye to how the application can inform clinicians about subtle differences in performance in order to better inform treatment recommendations as normal aging and disease progression exacerbate symptoms. However, our application confers benefit over prior Wacom applications in being widely accessible from any $\mathrm{WiFi}$ connected, mobile touchscreen device.

\section{Acknowledgments}

Funding: All authors were supported by Thomas Jefferson University institutional funds. Publication made possible in part by support from the Thomas Jefferson University Open Access Fund.

\section{Footnote}

Reporting Checklist: The authors have completed the MDAR reporting checklist. Available at https://dx.doi. org/10.21037/mhealth-21-16

Peer Review File: Available at https://dx.doi.org/10.21037/ mhealth-21-16

Conflicts of Interest: All authors have completed the ICMJE uniform disclosure form (available at https://dx.doi. org/10.21037/mhealth-21-16). The authors have no conflicts of interest to declare.

Ethical Statement: The authors are accountable for all aspects of the work in ensuring that questions related to the accuracy or integrity of any part of the work are appropriately investigated and resolved. The study was conducted in accordance with the Declaration of Helsinki (as revised in 2013). The study was exempted by the Thomas Jefferson University Institutional Review Board (Control \#20E.990) and informed consent was taken from all 
individual participants.

Open Access Statement: This is an Open Access article distributed in accordance with the Creative Commons Attribution-NonCommercial-NoDerivs 4.0 International License (CC BY-NC-ND 4.0), which permits the noncommercial replication and distribution of the article with the strict proviso that no changes or edits are made and the original work is properly cited (including links to both the formal publication through the relevant DOI and the license). See: https://creativecommons.org/licenses/by-nc-nd/4.0/.

\section{References}

1. Schuhmayer N, Weber C, Kieler M, et al. Task-dependent variability of Essential Tremor. Parkinsonism Relat Disord 2017;41:79-85.

2. Haubenberger D, Kalowitz D, Nahab FB, et al. Validation of digital spiral analysis as outcome parameter for clinical trials in essential tremor. Mov Disord 2011;26:2073-80.

3. Lin PC, Chen KH, Yang BS, et al. A digital assessment system for evaluating kinetic tremor in essential tremor and Parkinson's disease. BMC Neurol 2018;18:25.

4. Solé-Casals J, Anchustegui-Echearte I, Marti-Puig P, et al. Discrete Cosine Transform for the Analysis of Essential Tremor. Front Physiol 2019;9:1947.

5. Louis ED, Yu Q, Floyd AG, et al. Axis is a feature of handwritten spirals in essential tremor. Mov Disord 2006;21:1294-5.

6. Wang S, Bain PG, Aziz TZ, et al. The direction of oscillation in spiral drawings can be used to differentiate distal and proximal arm tremor. Neurosci Lett 2005;384:188-92.

7. Louis ED, Gillman A, Boschung S, et al. High width variability during spiral drawing: further evidence of cerebellar dysfunction in essential tremor. Cerebellum 2012;11:872-9.

8. Hsu AW, Piboolnurak PA, Floyd AG, et al. Spiral analysis in Niemann-Pick disease type C. Mov Disord 2009;24:1984-90.

9. San Luciano M, Wang C, Ortega RA, et al. Digitized Spiral Drawing: A Possible Biomarker for Early Parkinson's Disease. PLoS One 2016;11:e0162799.

10. Hess CW, Hsu AW, Yu Q, et al. Increased variability in spiral drawing in patients with functional (psychogenic) tremor. Hum Mov Sci 2014;38:15-22.

11. Longstaff MG, Heath RA. Spiral drawing performance as an indicator of fine motor function in people with multiple sclerosis. Hum Mov Sci 2006;25:474-91.

12. DelMastro HM, Ruiz JA, Gromisch ES, et al. Quantification characteristics of digital spiral analysis for understanding the relationship among tremor and clinical measures in persons with multiple sclerosis. J Neurosci Methods 2018;307:254-9.

13. Miralles F, Tarongí S, Espino A. Quantification of the drawing of an Archimedes spiral through the analysis of its digitized picture. J Neurosci Methods 2006;152:18-31.

14. Kraus PH, Hoffmann A. Spiralometry: computerized assessment of tremor amplitude on the basis of spiral drawing. Mov Disord 2010;25:2164-70.

15. Miralles F, Tarongí S, Espino A. Quantitative evaluation of the drawing of a spiral on a paper. Mov Disord 2011;26:1369; author reply 1369-70.

16. Saunders-Pullman R, Derby C, Stanley K, et al. Validity of spiral analysis in early Parkinson's disease. Mov Disord 2008;23:531-7.

17. Wang H, Yu Q, Kurtis MM, et al. Spiral analysis-improved clinical utility with center detection. J Neurosci Methods 2008;171:264-70.

18. Elble RJ, Ellenbogen A. Digitizing Tablet and FahnTolosa-Marín Ratings of Archimedes Spirals have Comparable Minimum Detectable Change in Essential Tremor. Tremor Other Hyperkinet Mov (N Y) 2017;7:481.

19. Westin J, Dougherty M, Nyholm D, et al. A home environment test battery for status assessment in patients with advanced Parkinson's disease. Comput Methods Programs Biomed 2010;98:27-35.

20. Westin J, Ghiamati S, Memedi M, et al. A new computer method for assessing drawing impairment in Parkinson's disease. J Neurosci Methods 2010;190:143-8.

21. Westin J, Schiavella M, Memedi M, et al. Validation of a home environment test battery for supporting assessments in advanced Parkinson's disease. Neurol Sci 2012;33:831-8.

22. Sisti JA, Christophe B, Seville AR, et al. Computerized spiral analysis using the iPad. J Neurosci Methods 2017;275:50-4.

23. Ishii N, Mochizuki Y, Shiomi K, et al. Spiral drawing: Quantitative analysis and artificial-intelligencebased diagnosis using a smartphone. J Neurol Sci 2020;411:116723.

24. Ondo WG, Wang A, Thomas M, et al. Evaluating factors that can influence spirography ratings in patients 
with essential tremor. Parkinsonism Relat Disord 2005;11:45-8.

25. Pullman SL. Spiral analysis: a new technique for measuring tremor with a digitizing tablet. Mov Disord

doi: $10.21037 /$ mhealth-21-16

Cite this article as: Magee R, Yang B, Ratliff J. Trsper: a webbased application for Archimedes spiral analysis. mHealth 2022;8:3.
1998;13 Suppl 3:85-9.

26. Dorsey ER, Glidden AM, Holloway MR, et al.

Teleneurology and mobile technologies: the future of neurological care. Nat Rev Neurol 2018;14:285-97. 


\section{Supplementary}

A

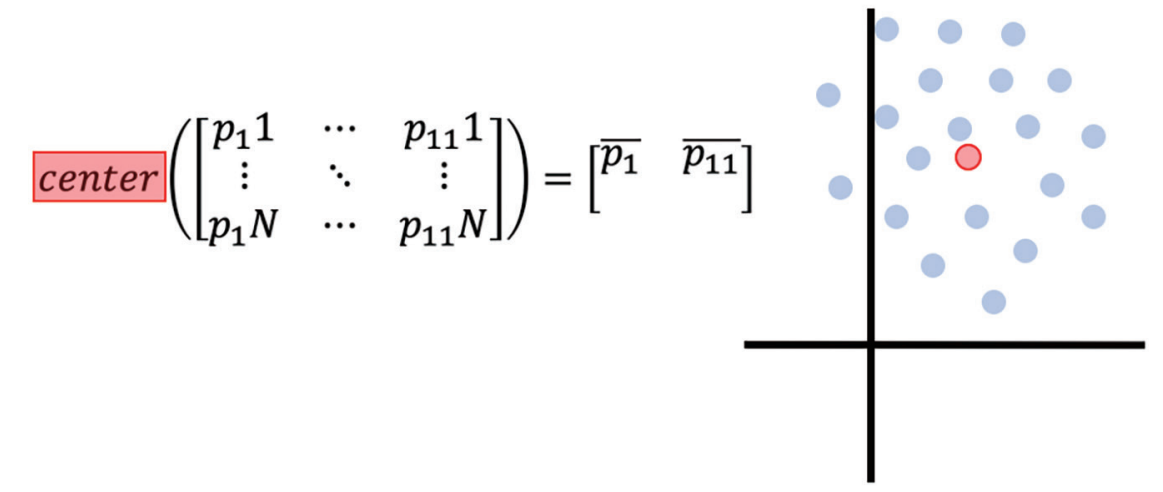

B

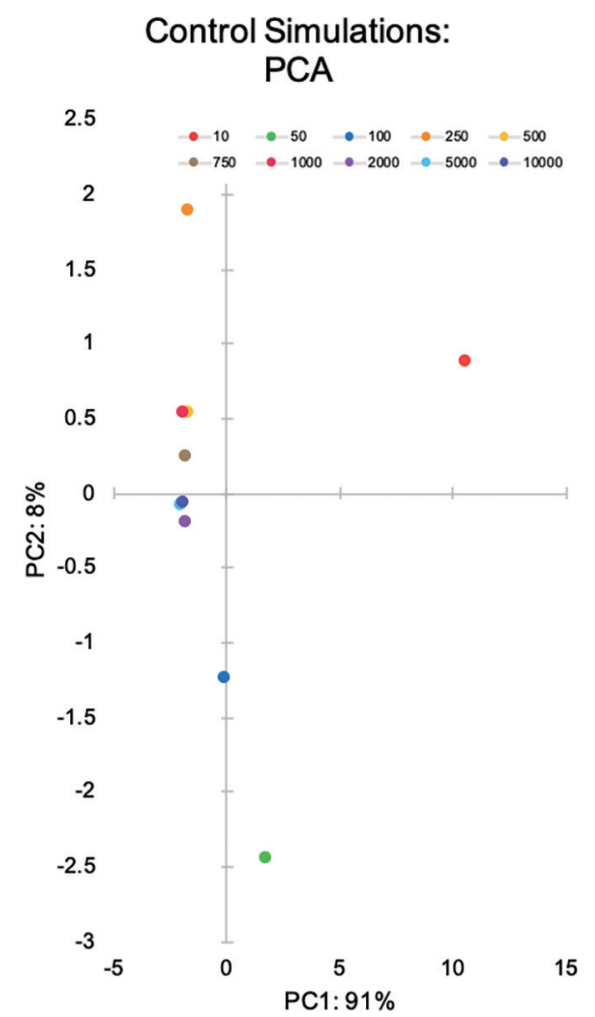

C

Control Simulations:

Distance to Centroid

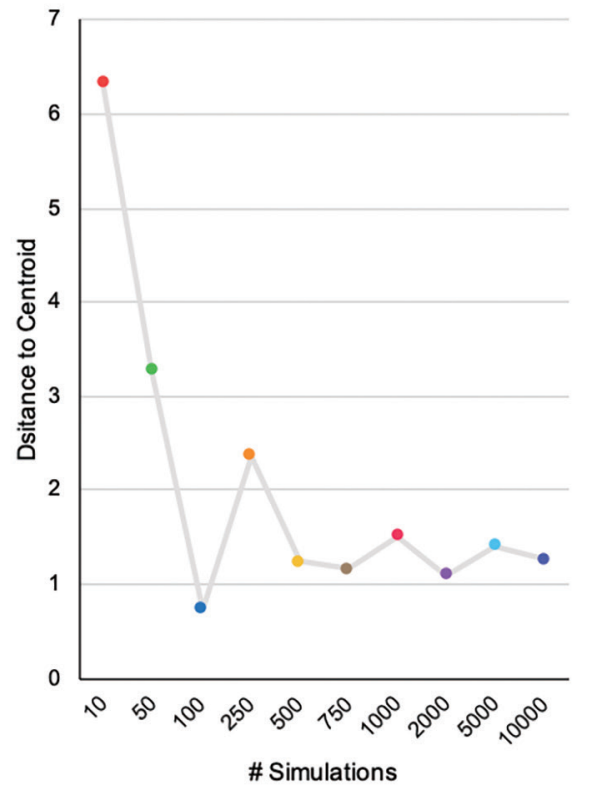

15

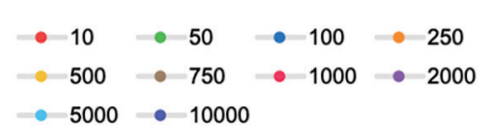

Figure S1 Ideal number of simulations. (A) Visual representation of the centroid of a group of points. (B) We simulated n=10, 50, 100, 250, 500, 750, 1,000, 2,000, 5,000, and 10,000 ideal Archimedean spirals with dithering, calculated the 11 metrics, and found the centroid of each group. We then use principal components analysis (PCA) to plot these centroids. (C) After finding the centroid of the PCA, we plot the distance from each PCA point to the centroid, noting a plateau in distance to the center after $\mathrm{n}=1,000$. 